\title{
The Identification of Intrinsic Chloramphenicol and Tetracycline Resistance Genes in Members of the Bacillus cereus Group (sensu lato)
}

\author{
Helen Glenwright ${ }^{1}$, Susanne Pohl ${ }^{1}$, Ferran Navarro ${ }^{2}$, Elisenda Miro ${ }^{2}$, Guillermo Jiménez ${ }^{3}$, \\ Anicet R. Blanch ${ }^{4}$ and Colin R. Harwood ${ }^{1 *}$ \\ ${ }^{1}$ Centre for Bacterial Cell Biology, Institute for Cell and Molecular Biology, Newcastle University, Newcastle upon Tyne, UK, \\ ${ }^{2}$ Servei de Microbiologia, Hospital de la Santa Creu i Sant Pau, Institut d'Investigació Biomèdica Sant Pau, Barcelona, Spain, \\ ${ }^{3}$ Research and Development Department, Rubinum S.A., Rubí, Spain, ${ }^{4}$ Department of Microbiology, University of Barcelona, \\ Barcelona, Spain
}

OPEN ACCESS

Edited by:

Frank T. Robb,

University of Maryland, Baltimore,

USA

Reviewed by:

Asli Aslan,

Georgia Southern University, USA

Antonio Juárez,

University of Barcelona, Spain

Bärbel Ulrike Fösel,

German Collection of Microorganisms

and Cell Cultures (LG), Germany

*Correspondence:

Colin R. Harwood

colin.harwood@ncl.ac.uk

Specialty section:

This article was submitted to

Evolutionary and Genomic

Microbiology,

a section of the journal

Frontiers in Microbiology

Received: 05 August 2016 Accepted: 15 December 2016

Published: 04 January 2017

Citation:

Glenwright $H$, Pohl S, Navarro $F$

Miro E, Jiménez G, Blanch AR and Harwood CR (2017) The Identification of Intrinsic Chloramphenicol and

Tetracycline Resistance Genes in Members of the Bacillus cereus Group (sensu lato). Front. Microbiol. 7:2122.

doi: 10.3389/fmicb.2016.02122
Bacillus toyonensis strain BCT-7112 ${ }^{\top}$ (NCIMB 14858 ${ }^{\top}$ ) has been widely used as an additive in animal nutrition for more than 30 years without reports of adverse toxigenic effects. However, this strain is resistant to chloramphenicol and tetracycline and it is generally considered inadvisable to introduce into the food chain resistance determinants capable of being transferred to other bacterial strains, thereby adding to the pool of such determinants in the gastro-enteric systems of livestock species. We therefore characterized the resistance phenotypes of this strain and its close relatives to determine whether they were of recent origin, and therefore likely to be transmissible. To this end we identified the genes responsible for chloramphenicol (catQ) and tetracycline (tetM) resistance and confirmed the presence of homologs in other members of the B. toyonensis taxonomic unit. Unexpectedly, closely related strains encoding these genes did not exhibit chloramphenicol and tetracycline resistance phenotypes. To understand the differences in the behaviors, we cloned and expressed the genes, together with their upstream regulatory regions, into Bacillus subtilis. The data showed that the genes encoded functional proteins, but were expressed inefficiently from their native promoters. B. toyonensis is a taxonomic unit member of the Bacillus cereus group (sensu lato). We therefore extended the analysis to determine the extent to which homologous chloramphenicol and tetracycline resistance genes were present in other species within this group. This analysis revealed that homologous genes were present in nearly all representative species within the $B$. cereus group (sensu lato). The absence of known transposition elements and the observations that they are found at the same genomic locations, indicates that these chloramphenicol and tetracycline resistance genes are of ancient origin and intrinsic to this taxonomic group, rather than recent acquisitions. In this context we discuss definitions of what are and are not intrinsic genes, an issue that is of fundamental importance to both Regulatory Authorities, and the animal feed and related industries.

Keywords: Bacillus toyonensis, gene knockouts, intrinsic antibiotic resistance, Bacillus cereus, quantitative PCR 


\section{INTRODUCTION}

The Bacillus cereus group (sensu lato) forms an independent branch within the Bacillus genus and currently comprises eight closely related and validated species: $B$. cereus (sensu stricto), B. anthracis, B. thuringiensis, B. mycoides, B. pseudomycoides, B. weihenstephanensis, B. cytotoxicus, and B. toyonensis (Skerman et al., 1980; Lechner et al., 1998; Guinebretière et al., 2013; Jiménez et al., 2013). Three additional new species, $B$. gaemokensis, B. manliponensis, and B. bingmayongensis, have been recently reported to belong to $B$. cereus group, but their names are not currently validly published (Jung et al., 2010, 2011; Liu et al., 2014, 2015).

Until relatively recently, understanding the identification and phylogenetic relationships within the $B$. cereus group (sensu lato) has proved challenging because: (a) traditionally, taxonomic distinctions among this group have relied heavily on a small number of phenotypic traits that include, in some cases, genes encoded on plasmids (e.g., anthrax toxin, insecticidal toxins, immune-evading capsules) and morphological features (B. mycoides; Rasko et al., 2004); (b) 16S rRNA gene sequence data is not sufficient to differentiate bacteria within the group due to its high degree of conservation (Bavykin et al., 2004).

In a taxonomic study to determine whether $B$. toyonensis strain BCT-7112 ${ }^{\mathrm{T}}$, previously known as B. cereus var. toyo $i$, strain BCT-7112, was a new species within the $B$. cereus group, a polyphasic approach was performed in which both phenotypic and genotypic traits were analyzed (Jiménez et al., 2013). Taken together, the results indicated that strain BCT-7112 ${ }^{\mathrm{T}}$ did indeed represent a new species for which the name B. toyonensis sp. nov. was proposed and approved, with BCT-7112 ${ }^{\mathrm{T}}$ (=CECT $876^{\mathrm{T}}$; $=$ NCIMB $14858^{\mathrm{T}}$ ) as the type strain (Oren and Garrity, 2014). The characteristics that distinguished strain BCT-7112 ${ }^{\mathrm{T}}$ from other $B$. cereus species were also shared by 10 other strains (Jiménez et al., 2013).

In a more recent taxonomic study, the affiliations of strains in the B. cereus group were established using a Genome BLAST Distance Phylogeny (GBDP) approach (Liu et al., 2015). This separated the 224 analyzed strains into 30 clusters; eleven known species-level clusters and 19 potentially novel species. In this analysis the 11 strains previously identified as belonging to the B. toyonensis taxonomic unit (Jiménez et al., 2013) formed a distinct cluster (BCG09) but was expanded to nineteen strains by the inclusion of eight additional strains. In the same study, an identical cluster was identified using a rapid typing method based on the $p y c A$ gene and, in separate studies, by multi-locus sequence analysis (MLSA) and whole-genome single nucleotide polymorphism (SNP)-based phylogeny (Böhm et al., 2015).

B. toyonensis BCT-7112 $2^{\mathrm{T}}$ has been widely used for more than 30 years as the active ingredient of Toyocerin ${ }^{\circledR}$, an additive used in animal nutrition and its non-toxigenic nature has been shown in various studies (Williams et al., 2009; Trapecar et al., 2011; Blanch et al., 2014). However, it is resistant to chloramphenicol and tetracycline, a trait that is generally considered inadvisable for introduction into the food chain if associated with mobile genetic elements (MGEs). We therefore set out to identify the genes responsible for these resistance phenotypes and to establish whether they were intrinsic or recent acquisitions associated with horizontal gene transfer. Unexpectedly, we not only observed homologous genes in closely related strains, but in virtually all members of the $B$. cereus group (sensu lato), irrespective of their reported susceptibility to these antibiotics. We discuss our findings in the context of regulations governing the animal feed and related industries.

\section{METHODS}

\section{Strains and Culture Conditions}

The strains and plasmids used in this study, together with their sources, are shown in Table 1. When required, the following antibiotic concentrations were used: for E. coli, $100 \mu \mathrm{g} / \mathrm{ml}$ ampicillin and $12.5 \mu \mathrm{g} / \mathrm{ml}$ chloramphenicol; for Bacillus strains, $1 \mu \mathrm{g} / \mathrm{ml}$ erythromycin, $100 \mu \mathrm{g} / \mathrm{ml}$ spectinomycin, and for chloramphenicol and tetracycline concentrations ranged from 1 to $32 \mu \mathrm{g} / \mathrm{ml}$, as indicated in the text.

Minimum inhibitory concentration (MIC) experiments were carried out in accordance with the European Food Safety Authority's (EFSA) "Guidance on the assessment of bacterial antimicrobial susceptibility” (EFSA-FEEDAP, 2012) using the microdilution method. Solutions of the required antibiotics $(0$, $1,2,4,8,16$, and $32 \mu \mathrm{g} / \mathrm{ml}$ ) were made up in Brain Heart Infusion broth and $200 \mu \mathrm{l}$ added to wells in a 96-well microtitre plate (MTP). The wells were inoculated with $20 \mu \mathrm{l}$ of the test cultures, prepared from fresh colonies grown on Brain Heart Infusion agar. The MTP was incubated in a FLUOstar Optima plate reader for $18 \mathrm{~h}$ at $37^{\circ} \mathrm{C}$ with vigorous shaking, monitoring Optical Density (OD) at $600 \mathrm{~nm}$. The experiments were carried out a minimum of three times and the representative data show on a linear rather than logarithmic graph to make it easier to compare growth profiles. The MIC was determined as the lowest concentration of the antibiotic for which no growth was detected.

\section{Construction of tetM and catQ Knockout Strains}

In order to identify the genes responsible for tetracycline and chloramphenicol resistance in the genome of $B$. toyonensis BCT-7112 ${ }^{\mathrm{T}}$, a gene replacement strategy was employed. This involved the replacement of the target gene with a spectinomycin gene by a reciprocal recombination event. Briefly, two DNA knockout cassettes of $\sim 3 \mathrm{~kb}$ were synthesized (DNA 2.0, Newark, California) and cloned into the vector pUTE583 (Koehler et al., 1994) to generate pUTE $\Delta$ tetM and pUTE $\Delta$ catQ. Each synthesized fragment consisted of three components, a $\sim 1000$ bp region upstream of the target gene, a 947-bp spectinomycin resistance fragment and a $\sim 1000$-bp regions downstream of the target cassette. In the case of the tet $M$ knockout cassette, a 1102-bp upstream fragment of the B. toyonensis BCT-7112 chromosome (nucleotides 305983-307085) was fused to the 947bp spectinomycin resistance fragment, which in turn was fused to the 907-bp downstream fragment of the B. toyonensis BCT$7112^{\mathrm{T}}$ chromosome (nucleotides 309075-309983; Figure 1A). The catQ knockout cassette was similarly constructed with a 1069-bp upstream fragment (nucleotides 4932601-4933670) and 1219 downstream fragment (nucleotides 4933976-4935195) of 
TABLE 1 | Strains and plasmids used in this study.

\begin{tabular}{|c|c|c|}
\hline Strains & Trait or relevant genotype* & Source/Reference* \\
\hline ATCC $14579^{\top}$ & & ATCC \\
\hline ATCC 10792 & & ATCC \\
\hline \multicolumn{3}{|l|}{ Bacillus toyonensis } \\
\hline BCT-7112 & B. toyonensis type strain, $\mathrm{Tc}^{\mathrm{R}}, \mathrm{Cm}^{\mathrm{R}}$ & Rubinum S.A, Spain \\
\hline BCT-7112 $\Delta$ cat & BCT-7112 ${ }^{\top}$ with the tetM gene replaced with a spectinomycin resistance gene, $\mathrm{Tc}^{\mathrm{R}}, \mathrm{Sp}^{\mathrm{R}}, \mathrm{Cm}^{\mathrm{S}}$ & This study \\
\hline Rock1-3 & B. toyonensis strain isolated from soil in Rockville, Maryland. Previously B. cereus Rock1-3 & $\begin{array}{l}\text { US Naval Medical Research Center } \\
\text { Zwick et al., } 2012\end{array}$ \\
\hline Rock3-28 & B. toyonensis strain isolated from soil in Rockville, Maryland. Previously B. cereus Rock3-28 & $\begin{array}{l}\text { US Naval Medical Research Center; } \\
\text { Zwick et al., } 2012\end{array}$ \\
\hline BCT-7112(plGR1) & BCT-7112 ${ }^{\top}$ with plGR, $\mathrm{Tc}^{\mathrm{R}}, \mathrm{Cm}^{\mathrm{R}}, \mathrm{Em}^{\mathrm{R}}$ & This study \\
\hline \multicolumn{3}{|l|}{ Bacillus subtilis } \\
\hline 168 & $\operatorname{trpC2}$ & $\begin{array}{l}\text { Institute Pasteur, Paris; } \\
\text { Anagnostopoulos and Spizizen, } 1961\end{array}$ \\
\hline 168tetM7112 & 168 with tetM from BCT-7112 ${ }^{\top}, \mathrm{TC}^{\mathrm{R}}$ & This study \\
\hline 168tetM1-3 & 168 with tetM from Rock1-3, $\mathrm{Tc}^{\mathrm{R}}$ & This study \\
\hline 168tetM3-28 & 168 with tetM from Rock3-28, $\mathrm{Tc}^{\mathrm{R}}$ & This study \\
\hline 168catQ7112 & 168 with catQ from BCT-7112 ${ }^{\top}, \mathrm{Cm}^{R}$ & This study \\
\hline 168catQ1-3 & 168 with catQ from Rock1-3, $\mathrm{Cm}^{R}$ & This study \\
\hline 168catQ3-28 & 168 with catQ from Rock3-28, $\mathrm{Cm}^{R}$ & This study \\
\hline \multicolumn{3}{|l|}{ Escherichia coli } \\
\hline $\mathrm{DH} 5 \alpha$ & huA2 lac(del)U169 phoA glnV44 $\Phi 80^{\prime}$ lacZ(del)M15 gyrA96 recA1 relA1 endA1 thi-1 hsdR & Thermo Fisher Scientific \\
\hline pDR111 & B. subtilis integration/expression vector, $A p^{R}\left(E\right.$. coli), $S p^{R}$ (B. subtilis), amyE $\Delta$, lacl, $P_{\text {hyper-spank }}$ & R. Daniel, Newcastle University \\
\hline pDRtetM7112 & pDR111 with tetM from BCT-7112 ${ }^{\top}, \mathrm{Ap}^{R}, \mathrm{Sp}^{\mathrm{R}}, \mathrm{Tc}^{\mathrm{R}}$ & This study \\
\hline pDRtetM1-3 & pDR111 with tetM from Rock1-3, $A p^{R}, S^{R}, T_{c}^{R}$ & This study \\
\hline pDRtetM3-28 & pDR111 with tetM from Rock3-28, $\mathrm{Ap}^{R}, \mathrm{Sp}^{\mathrm{R}}, \mathrm{Tc}^{\mathrm{R}}$ & This study \\
\hline pDRcatQ7112 & pDR111 with catQ from BCT-7112 ${ }^{T}, A p^{R}, S p^{R}, T_{c}^{R}$ & This study \\
\hline pDRcatQ1-3 & pDR111 with catQ from Rock1-3, $A p^{R}, S p^{R}, T_{c}^{R}$ & This study \\
\hline pDRcatQ3-28 & pDR111 with catQ from Rock3-28, $A p^{R}, S^{R}, T_{c}^{R}$ & This study \\
\hline
\end{tabular}

${ }^{*} A T C C$, American Type Culture Collection; $A p^{R}$, ampicillin resistance; $\mathrm{Cm}^{R}$, chloramphenicol resistance; $\mathrm{Em}^{R}$, erythromycin resistance; $T c^{R}$, tetracycline resistance; Sp ${ }^{R}$, spectinomycin resistance.

the B. toyonensis BCT-7112 $2^{\mathrm{T}}$ chromosome, separated by the 947-bp spectinomycin resistance fragment (Figure 1B).

The bifunctional vector, pUTE853, is able to replicate in $E$. coli and members of the $B$. cereus group, but is lost from the latter at $37^{\circ} \mathrm{C}$ in the absence of antibiotic selection. The knockout plasmids, pUTE $\Delta$ tetM and pUTE $\Delta$ catQ, were first passaged through a dam-minus E. coli strain (GM48) and then electro-transformed into B. toyonensis BCT-7112 ${ }^{\mathrm{T}}$, selecting for erythromycin resistance at $30^{\circ} \mathrm{C}$ (Koehler et al., 1994). The transformants were grown in the presence of erythromycin for 2 days, diluting 1:500 at the start and end of the day with fresh medium, and then shifted to $37^{\circ} \mathrm{C}$ for 2 days in the presence of spectinomycin, again diluting 1:500 at the start and end of the day with fresh medium. Samples were plated onto BHI agar containing spectinomycin, incubated overnight at $37^{\circ} \mathrm{C}$, and individual colonies checked for erythromycin 


\section{A tet $M$ knockout (KO) strain}
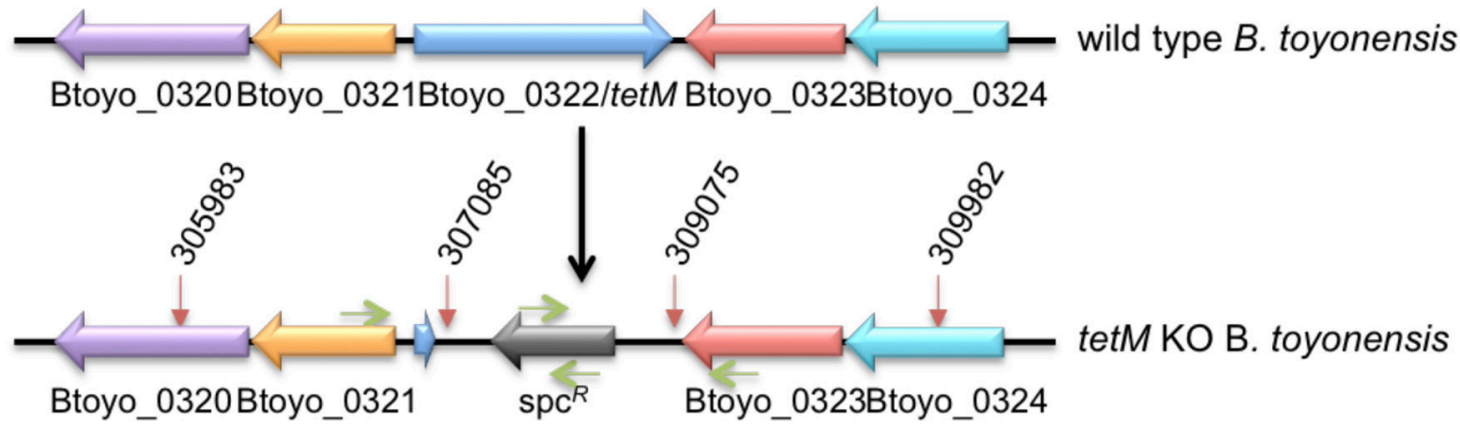

B catQ knockout $(\mathrm{KO})$ strain

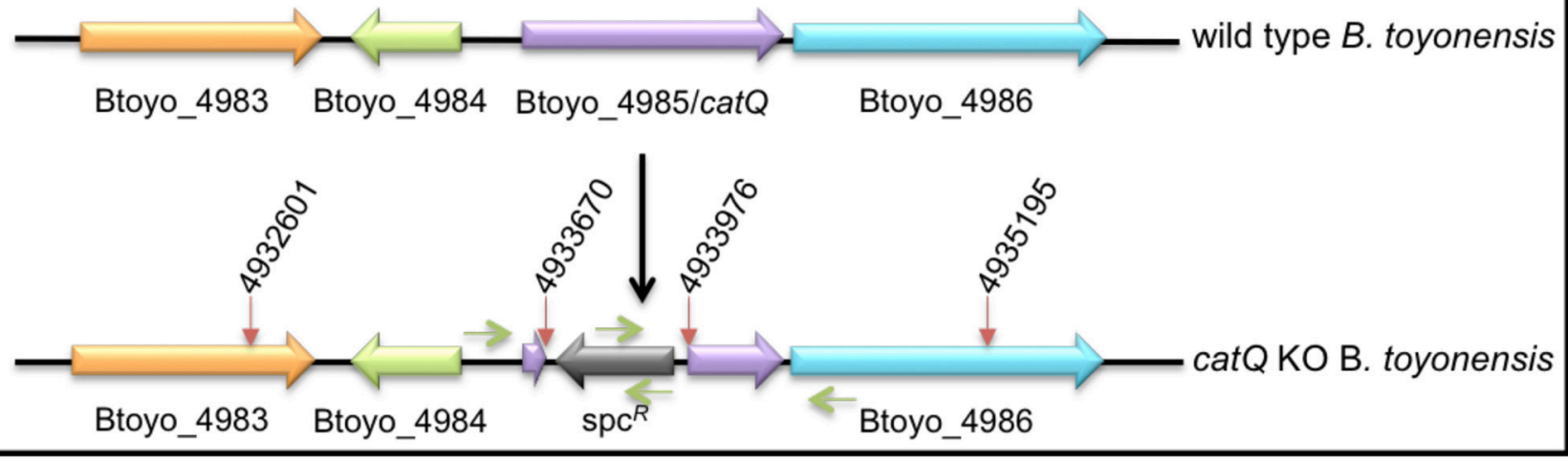

FIGURE 1 | Regions of the B. toyonensis BCT-7112 ${ }^{\mathbf{T}}$ genome containing (A) tetracycline (Btoyo_0322, tetM) and (B) chloramphenicol (Btoyo_4985, catQ) resistance genes. The lower images show the locations (red arrows) of the upstream and downstream fragments and the spectinomycin resistance (spc ${ }^{R}$ ) gene that were used to replace the Btoyo_0322 or Btoyo_4985 genes by reciprocal recombination events, generating the tetM or catQ knockout strains BCT-7112 $\Delta$ tet and BCT-7112 $\Delta$ cat. The green arrows show the approximate locations of the primers used to sequence over the knockout regions.

sensitivity and spectinomycin resistance, confirming the loss of the plasmid and integration of the knockout cassette into the chromosome. In each case the target antibiotic resistance phenotype was lost, tetracycline resistance in the case of pUTE $\Delta$ tetM and chloramphenicol resistance in the case of pUTE $\Delta$ catQ, generating $B$. toyonensis knockout strains BCT$7112 \Delta$ tet and BCT-7112 $\Delta$ cat, respectively. The fidelity of the integration event was confirmed by sequencing over the region using primers within the flanking fragments and spectinomycin fragment (Figure 1).

\section{Construction of Strains with Additional Copies of the gerIC-nucB Intergenic Region}

Previous unpublished studies indicated an involvement of the gerIC-nucB intergenic region of plasmid pBCT77 as contributing to, or enhancing, the observed tetracycline and chloramphenicol resistances in B. toyonensis $\mathrm{BCT}-7112^{\mathrm{T}}$. The 2003-bp gerIC$n u c B$ intergenic region extends from nucleotide $75752-76974$ on the pBCT77 genome (NC_022782). In the first place we used primers 1pBCT77Fwd ( $5^{\prime}$-GCCCCTTTAAACTTCATTATGC$\left.3^{\prime}\right)$ and 4 pBCT77Rev (5'-CCCTTACCTTCAAGTATTCC-3') to confirm that pBCT77 was still present in both knockout strains. The intergenic DNA was then synthesized and cloned into the HindIII and XbaI sites of pUTE853 (DNA 2.0, Newark, California). The resulting vector, pIGR1, was transformed into E. coli strain $\mathrm{DH} 5 \alpha$ and its structure confirmed by restriction analysis and PCR. Following passage through the E. coli damminus strain GM48, pIGR1 was electro-transformed into $B$. toyonensis strains BCT-7112 ${ }^{\mathrm{T}}$, BCT-7112 $\Delta$ tet, BCT-7112 $\Delta$ cat, and Rock1-3, selecting for erythromycin resistant transformants at $37^{\circ} \mathrm{C}$. Erythromycin selection was maintained in all precultures, but not during MIC experiments.

\section{Construction $B$. subtilis Strains with tetM and catQ Genes from BCT-7112 ${ }^{\top}$, Rock1-3, and Rock3-28}

For expression in B. subtilis, the tet $M$, and catQ genes from BCT-7112 ${ }^{\mathrm{T}}$, Rock1-3, and Rock3-28 were first cloned into the bifunctional expression/integration vector pDR111 
(Britton et al., 2002). This vector has a replication origin for $E$. coli but not B. subtilis, and therefore can only persist in B. subtilis by integration into the amyE locus via front-end and back-end fragments of this gene. The intervening sequences include a spectinomycin resistance genes and a copy of lacI encoding the Lac repressor. Finally, downstream of a strong $\mathrm{P}_{\text {hyper-spank }}$ promoter is a multiple cloning site. The respective tet $M$ and catQ genes were PCR amplified from the B. toyonensis strains with SalI and SphI sites using the following primers: (i) for the tet $M$ genes of strains BCT-7112 ${ }^{\mathrm{T}}$ and Rock1-3 strains, tetMFwd1 (5'-GGCCGGGTCGACTGAGCATGAACTTGTCAAACTACC C-3') and tetMRev1 (5'- GGCCGGGCATGCGTAATAGAA ACACTTAAAGAAGTTGTTAGG-3') and strain Rock3-28, tetMFwd2 (5'- GGCCGGGTCGACTGAGCATGAACTTGT CAAATTACCC- $3^{\prime}$ ) and tetMRev2 (5'- GGCCGG-GCATGCGCGCCCTCTAGTAAGGAAAAGTATC-3'); (ii) for the catQ genes of strains BCT-7112 ${ }^{\mathrm{T}}$, Rock1-3, and Rock3-28, catQFwd1 (5' - GGCCGGGTCGACTATTAATAGTATGTGGATGGATTG C-3') and catQRev1 (5'- GGCCGGGCATGCCAATTTTAT ATAATTAAAAATTTGATATTACTTAAAGCC-3'). Following ligation and transformation initially into E. coli strain $\mathrm{DH} 5 \alpha$, the structures of the recombinant plasmids (pDRtetM7112, pDRtetM1-3, pDRtetM3-28, pDRcatQ7112, pDRcatQ1-3, and pDRcatQ3-28, see Table 1) were confirmed by digestion and then transformed into B. subtilis 168 by natural transformation (Anagnostopoulos and Spizizen, 1961). The resulting strains were: for the tetM constructs, B. subtilis strains 168tetM7112, 168tetM1-3, and 168tetM3-28; and for the catQ constructs, B. subtilis strains 168catQ7112, 168catQ1-3, and 168catQ3-28 (Table 1).

The growth parameters of $B$. subtilis with integrated copies of the tetM and catQ genes from B. toyonensis strains BCT-7112 ${ }^{\mathrm{T}}$, Rock1-3, and Rock3-28 were determined in the presence of various concentrations of tetracycline and chloramphenicol. Antibiotic solutions (0, 4, 8, 16, and $32 \mu \mathrm{g} / \mathrm{ml})$ were prepared in Brain Heart Infusion broth, prewarmed to $37^{\circ} \mathrm{C}$ and distributed to in $800 \mu \mathrm{l}$ amounts into a 48 -well FlowerPlate $^{\circledR}$ (m2pLabs, Baesweiler, Germany). The individual wells were inoculated to an $\mathrm{OD}_{600}$ of 0.05 with the required cultures (168tetM7112, 168tetM1-3, 168tetM3-28, 168catQ7112, 168catQ1-3, and 168catQ3-28), previously grown overnight in Brain Heart Infusion broth without antibiotics. The experiment was duplicated with one half of the cultures containing IPTG $(1 \mathrm{mM})$ to induced expression from the upstream $\mathrm{P}_{\text {hyper-spanc }}$ promoter and the other half relying on their cognate upstream regulatory regions. The cultures were incubated in a BioLector ${ }^{\circledR}$ micro bioreactor (m2pLabs) for $20 \mathrm{~h}$ at $37^{\circ} \mathrm{C}$, with a gain of 25 and shaking at $1500 \mathrm{rpm}$. The BioLector microreactor determines the optical density of the culture by measuring the scattered light signal using an excitation light beam with a wavelength of $620 \mathrm{~nm}$. The data is shown on a linear rather than logarithmic graph to make it easier to compare growth profiles.

\section{Visualization of Cells by Light Microscopy}

Overnight cultures in Müller Hinton Broth (MHB) were used to inoculate fresh broth to an $\mathrm{OD}_{600}$ of 0.05 . The cells were grown at $37^{\circ} \mathrm{C}$ with shaking until the $\mathrm{OD}_{600}$ reached 0.2 when chloramphenicol ( 2 or $32 \mu \mathrm{g} / \mathrm{ml}$ ) was added. Samples were taken every $60 \mathrm{~min}$ for visualization by phase contrast microscopy.

\section{Relative Quantification of tetM and catQ Genes Expression}

The expression of the tet $M$ and catQ genes was determined by a combination of Northern Blotting and quantitative (q) PCR, the former used to establish the transcript sizes the latter for quantitation.

For Northern blotting the Total RNA purification Kit (Norgen Biotek Corp) was used for the extraction of total RNA. The RNA integrity was determined by ensuring the $\mathrm{A}_{260 / 280}$ ratio was between 1.8 and 2.0 and by electrophoresis on a denaturing $1.2 \%$ agarose-formaldehyde gel. Northern Blot analysis was performed as described by Homuth et al. (1997). Digoxigenin (DIG) labeled catQ- and tetM-specific RNA probes were synthesized by in vitro transcription using T7 RNA polymerase and a DIG RNA Labelling Kit (Roche Diagnostics) from specific PCR products as templates. Primer sequences used to amplify the templates for the RNA probes were as follows: catQ-FWD: 5'-GTTGATATTACGATGATGCTAGAG AAAATAC-3', catQ-REV: 5'-CTAATACGACTCACTATAGGG AGACTTTCGGGAAGAGACCATGAAC-3'; tetM-FWD: $5^{\prime}-$ ATGAAACGAATGTGATTAAAGAAATTGGC-3', tetM-REV: 5'-CTAATACGACTCACTATAGGGAGAGGTGCTACTCGT TCCATACAATC-3'. The hybridization signal was developed using a digoxigenin-specific antibody conjugated to alkaline phosphatase (Roche Diagnostics) and CDP-Star (Thermo Fisher Scientific) as a chemiluminescence substrate. The images were captured on an ImageQuant LAS 4000 (GE Healthcare Life Sciences).

In the case of $\mathrm{qPCR}$, the unlabeled PCR primers and TaqMan ${ }^{\circledR}$ MGB probes $\left(5^{\prime}\right.$-FAM ${ }^{\text {TM }}$ dye labeled) were designed using the Assays-by-Design ${ }^{\mathrm{TM}}$ service (Applied Biosystems). The primers and probes used were as follows: for the tetM gene, TETP_Fwd (5'-CGGAAATGCAGTT CAAGCTTCTAC-3'), TETP_Rev (5'-AATTTTAATAT CACTCAGTCCCCACACTT- $\left.3^{\prime}\right)$, and TETP_probe $\left(5^{\prime}-\right.$ CTAGCGGAGAATTTT-3); for the catQ gene, CAT._Fwd ( $5^{\prime}-\mathrm{C}$ TAATGTTCATGGTCTCTTCCCGAAA-3'), CAT._Rev 5'-CT AGTCCAAGGTATCCCAGAAATCG-3'), and CAT_probe (5'-TTGGCGGAATATTTTC-3'); for the ${ }^{\prime}$; $y r B$ gene, GYRB_Fwd (5'-CAGCAATCGTGTCAATTAAACATCCAA-3'), GYRB_Rev (5'-CCGTAATCGTTCTCGCTTCACTATT-3'), and GYRB_probe (5'-ACGAAGACGAAACTTG-3').

The RNAprotect Bacteria Reagent (QIAGEN) was used to stabilize RNA by preventing the degradation of RNA transcripts and the induction of genes. The bacterial strains were cultured in Luria Bertani (LB) medium at $37^{\circ} \mathrm{C}$. Depending on the strain and species, the incubation time required to reach exponential phase was $2-3 \mathrm{~h}$ before harvesting the cells. RNA extraction was performed using the RNeasy Protect Bacteria MiniKit (QIAGEN), according to the manufacturer's instructions. The expression of the housekeeping gene $g y r B$ was used as baseline control for relative quantification. 
The quality and concentration of RNA was measured using a Nanodrop 1000 Spectrophotometer, as recommended in the high capacity cDNA reverse transcription kit (Applied Biosystems), and adjusted at $50 \mathrm{ng} / \mu \mathrm{l}$ for relative quantification and PCR normalization. The quantitative PCR was performed using the qPCR MasterMix No ROX (Eurogenetec) and the Custom TaqMan Gene Expression Assays system (Applied Biosystems). The calculation of the relative expression ratios of the target genes was based on the mathematical model of Pfaffl (2001).

\section{Bioinformatical Analyses}

BLASTp was used to identify homologs of the B. toyonensis $7112^{\mathrm{T}}$ CatQ and TetM proteins among sequences in the NCBI genome database (Altschul et al., 1990). The data was downloaded in CVS format and uploaded into Excel (Tables S1, S2). The data shows the Reference Sequence IDs, individual Sequence IDs, the description of the protein in the annotation file, the bacterial source, alignment score, $E$-value and \% identity. The BLASTp data was also used to construct Neighbor Joining trees of homologs of the B. toyonensis BCT-7112 ${ }^{\mathrm{T}}$ CatQ and TetM proteins in the NCBI genome database. The algorithm used (Saitou and Nei, 1987) produces an un-rooted tree. The maximum allowed fraction of mismatched bases between any pair of sequences was 0.85 . The evolutionary distance between two sequences was modeled as the expected fraction of amino acid substitutions per site, based on the fraction of mismatched amino acids in the aligned region (Grishin, 1995).

\section{RESULTS}

\section{Characterization of Resistance Phenotypes and the Identification of Resistance Genes}

Minimum inhibitory concentrations (MICs) of B. toyonensis BCT- $7112^{\mathrm{T}}$ for tetracycline and chloramphenicol were, at 16 and $32 \mu \mathrm{g} / \mathrm{ml}$ respectively, higher than the resistance break points $(\leq 8 \mu \mathrm{g} / \mathrm{ml})$ identified by the European Committee on Antimicrobial Susceptibility Testing (Kahlmeter et al., 2006). Consequently B. toyonensis BCT- $7112^{\mathrm{T}}$ is designated as being resistant to both of these antibiotics. Analysis of the annotated B. toyonensis BCT-7112 ${ }^{\mathrm{T}}$ genome sequence (NCBI; CP006863), and plasmids pBCT77 (CP006864) and pBCT8 (CP006865), revealed the presence of genes encoding putative tetracycline and chloramphenicol resistance proteins.

The most common tetracycline resistance mechanisms are efflux pumps and ribosome-protection proteins (Wilson, 2014). The B. toyonensis BCT- $7112^{\mathrm{T}}$ genome encodes two putative tetracycline resistance proteins, one an efflux protein and the other a putative ribosome-protection protein. Btoyo_4389 (nucleotides 4295949-4297223) is annotated as encoding a tetracycline resistance protein of the Major Facilitator Superfamily (MFS, cd06174). The MFS is a large and diverse group of secondary transporters that includes uniporters, symporters, and antiporters. MFS proteins facilitate transport across the cytoplasmic membrane of various substrates, including ions, sugar phosphates, antibiotics, amino acids, and peptides.
MFS transporters are common among soil-living organisms and, without specific experimental evidence, it can be difficult to identity their substrates. BLAST analysis revealed that homologs of Btoyo_4389 are found extensively among members of the $B$. cereus group but are labeled, in decreasing order of frequency, "MFS transporter," "tetracycline resistance protein," major facilitator protein," and "putative multidrug resistance protein." It is not clear what, if any, evidence there is for annotating this protein specifically as a "tetracycline resistance protein" rather than as a "MFS transporter," illustrating a limitation of automated annotation programs.

Btoyo_0322 (nucleotides 307023-308966) is a putative ribosome protection protein with a conserved TetM-like family domain (cd04168). TetM proteins exhibit $\sim 45 \%$ similarity with elongation factor G (EF-G) proteins. They have conserved nucleotide-binding motifs and are members of the translation factor superfamily of GTPases that bring about the release of tetracycline from the ribosome in a GTP-dependent manner (Dönhöfer et al., 2012).

Several genes are annotated as encoding a TetR-like protein. TetR proteins are a class of transcriptional regulators with helix-turn-helix motifs, and are so annotated because the first representative of this class was responsible for the regulation of a MFS transporter mediating tetracycline resistance on transposon Tn10 (Beck et al., 1982). The association with tetracycline is therefore historic.

Analysis of the B. toyonensis BCT-7112 ${ }^{\mathrm{T}}$ genome reveals the presence of two genes encoding putative chloramphenicol acetyltransferases (Cat) that inactivate chloramphenicol by acetylation. Btoyo_3723 (nucleotides 3655712-3656095), at 127 residues in length, is significantly shorter than other chloramphenicol acetyltransferases ( $\sim 220$ residues) and is likely to have been truncated during evolution. Although, the active site is present, it is unlikely that this gene encodes an active enzyme. In contrast, Btoyo_4985 (nucleotides 4933576-4934223) encodes a full-length CatQ-like chloramphenicol acetyltransferase.

A previous unpublished transposon mutagenesis study (EFSA-FEEDAP, 2014; M Kato, Science Tanaka, Japan, personal communication) identified a region on pBCT77, between $n u c B$ (Btoyo_5072 [nucleotides 74482-74916]) and gerIC (Btoyo_4992 [nucleotides 250-858]) that had a minor influence on reducing the MICs of tetracycline and chloramphenicol in B. toyonensis BCT-7112 ${ }^{\mathrm{T}}$. Two of the three genes in this region encode proteins of unknown function (Btoyo_5073 [nucleotides 74939-75190]; Btoyo_5074 [nucleotides 75202-75432]), while the third gene (Btoyo_5075 [nucleotides 75457-76029]) encodes a phage-type site-specific recombinase. There were no genes encoding known antibiotic resistance genes in this region, elsewhere on this pBCT77 or on the smaller plasmid, pBCT8.

\section{Identification of the Genes Encoding Tetracycline and Chloramphenicol Resistances}

Btoyo_0322 and Btoyo_4389 were tentatively identified as encoding tetracycline resistance proteins, the former a TetMlike protein, the later a MFS-like transporter. Because of 
limitations in the identification of MFS substrates and the higher likelihood that Btoyo_4389 was incorrectly annotated, we focused on Btoyo_0322. The tetM gene encoded by Btoyo_0322 was replaced with a spectinomycin resistance gene (Section Construction of tetM and catQ Knockout Strains) and the resulting knockout strain (BCT-7112 $\Delta$ tet) was spectinomycin and chloramphenicol resistant, but erythromycin and tetracycline susceptible. The authenticity of the integration event was confirmed by sequencing across the entire region (see Figure 1A). The data confirmed that Btoyo_0322 was excised from the chromosome and that this gene was solely responsible for the observed tetracycline resistance phenotype of B. toyonensis strain BCT-7112 ${ }^{\mathrm{T}}$.

A similar approach was used to identify the gene responsible for chloramphenicol resistance. Of the two putative chloramphenicol resistance genes identified in the genome of BCT-7112 ${ }^{\mathrm{T}}$, Btoyo_3723, and Btoyo_4985, the later was chosen as the former appeared to have been truncated. The gene replacement strategy described in Section Construction of tet $M$ and catQ Knockout Strains was employed, involving the replacement of Btoyo_4985 with a spectinomycin resistance gene. The resulting knockout strain (BCT-7112 $\Delta$ cat), was spectinomycin and tetracycline resistant, but erythromycin and chloramphenicol susceptible. The authenticity of the integration event was again confirmed by sequencing across the entire region (see Figure 1B). The data confirmed that Btoyo_4985 was solely responsible for the observed chloramphenicol resistance phenotype of B. toyonensis strain BCT-7112 ${ }^{\mathrm{T}}$.

Following their identification, the \%GC contents of Btoyo_0332 and Btoyo_4985 were determined to be 37.3 and $29.8 \%$, respectively, while the genome average of $B$. toyonensis BCT-7112 ${ }^{\mathrm{T}}$ was $35.6 \%$. Using a 1000 -bp window and a standard deviation cut-off value of 2.5 , neither the $\% \mathrm{GC}$ of these coding sequences, nor a 3500-bp regions either side, were found to be significantly different from the running average, indicating that Btoyo_0332 and Btoyo_4985 are not recent acquisitions.

\section{The Presence of Homologs of tetM (Btoyo_322) and catQ (Btoyo_4985) Genes in Other Member of the $B$. cereus sensu lato Group}

In light of the recent studies revising the taxonomic status of the B. cereus group (sensu lato) (Jiménez et al., 2013; Böhm et al., 2015; Liu et al., 2015), we used BLASTp (Altschul et al., 1990) to search for homologs of the BCT-7112 ${ }^{\mathrm{T}}$ tet $M$ and cat $Q$ genes in the other 18 strains within the $B$. toyonensis taxonomic unit. The data (Table 2) clearly show that homologs of TetM are present in all strains of $B$. toyonensis while homologs of CatQ are present in all but one strain. The failure to detect CatQ in B. toyonensis VD115 is most likely to be due to the absence of the relevant region in the partial genome sequence, which is $3884 \mathrm{k}$ in length compare with $4940 \mathrm{k}$ for B. toyonensis BCT-7112 ${ }^{\mathrm{T}}$.

In the case of TetM, just under half of the B. toyonensis strains encode proteins with identical sequences (Table 2). The most variable TetM protein is encoded by B. toyonensis VD148, which shows $90 \%$ identity and $95 \%$ similarity with that of strain
BCT-7112 ${ }^{\mathrm{T}}$. Twelve of the B. toyonensis strains encode CatQ proteins that are identical to that of strain BCT-7112 ${ }^{\mathrm{T}}$, while the remaining strains exhibit CatQ proteins with $99 \%$ identity. Moreover, the tet $M$ and catQ genes are located in the same genomic neighborhoods in all of the B. toyonensis strains. Taken together, the data indicate that the tet $M$ and cat $Q$ genes are ancient genomic components of the B. toyonensis taxonomic unit rather than having been acquired recently by horizontal gene transfer.

The NCBI genomes database contains whole genomes, chromosomes, scaffolds and contiguous fragments (contigs) of $B$. anthracis, B. bombysepticus, B. cereus sensu stricto, B. mycoides, $B$. thuringiensis, B. toyonensis, B. weihenstephanensis, and $B$. wiedmannii (Table 3). In order to extend the homology study to other members of the B. cereus sensu lacto, BLASTp was used to search for homologs of the B. toyonensis BCT-7112 ${ }^{\mathrm{T}}$ TetM and CatQ proteins with a similarity score of $85 \%$ or greater (Table 4). All of the strains identified as having homologs of these TetM and CatQ proteins were members of the B. cereus group (sensu lato): no strains outside this group appeared in the analysis. Homologs of both the TetM and CatQ were found in all eight members of the B. cereus group shown in Table 3. Genes encoding these proteins were absent from $B$. cytotoxicus and B. pseudomycoides, although this could be due either to the small number of DNA sequences available for these species or to changes in their taxonomic status. A list of sequences analyzed for homology with the TetM and CatQ proteins is given in Tables S1, S2, respectively.

A total of 369 homologs of TetM were identified in the NBCI database and widely distributed among species of the $B$. cereus group, broadly reflecting the frequency of complete genomes and chromosomes in the database (Table 3). In the case of CatQ, 381 homologs were found, and with a distribution that was similar to that of TetM. The relationships between these TetM and CatQ sequences, in the form of Neighbor Joining trees, are shown in Figures S1, S2. In both cases the trees exclusively contain homologous proteins from other members of the $B$. cereus group (sensu lato), confirming the ancient origin of these proteins in this taxonomic clade.

\section{Tetracycline and Chloramphenicol Resistance Profiles of the Strains within the B. toyonensis Taxonomic Unit}

B. toyonensis BCT-7112 $2^{\mathrm{T}}$ is phenotypically resistant to tetracycline and chloramphenicol, and this study identified the genes responsible, namely tetM (Btoyo_0322) and catQ (Btoyo_4985). However, eight other B. toyonensis strains are phenotypically susceptible to tetracycline and chloramphenicol, despite encoding TetM and CatQ proteins that are homologous to those strain BCT-7112 ${ }^{\mathrm{T}}$ (EFSA-FEEDAP, 2014; L. Mocé, Eurofins Biolab, personal communication). We therefore addressed the question of why other members of the $B$. toyonensis taxonomic unit that encode either identical (strain Rock1-3) or similar (strain Rock3-28) resistance determinants do not exhibit the same resistance phenotypes. Both strains are susceptible to tetracycline and chloramphenicol (MIC $1 \mu \mathrm{g} / \mathrm{ml}$ ) 
TABLE 2 | The percentage identity and similarity of proteins encoded by homologs of the tetM and catQ genes of $B$. toyonensis BCT-7112 ${ }^{\top}$ among other members of the $B$. toyonensis taxonomic unit.

\begin{tabular}{|c|c|c|c|}
\hline Strain & GenBank Accession $N^{\circ}$ & $\begin{array}{l}\% \text { Identity/Similarity to } \\
\text { B. toyonensis BCT-7112 TetM }\end{array}$ & $\begin{array}{l}\% \text { Identity/Similarity to } \\
\text { B. toyonensis BCT-7112 CatQ }\end{array}$ \\
\hline B. toyonensis BCT- $7112^{\top}$ & СР006863.1 & $100 / 100$ & 100/100 \\
\hline B. toyonensis MC28* & СР003687.1 & $94 / 97$ & $100 / 100$ \\
\hline B. toyonensis Rock3-28* & CM000730.1 & 93/96 & $100 / 100$ \\
\hline B. toyonensis VD148* & JH792156.1 & 90/95 & $100 / 100$ \\
\hline B. toyonensis BAG2O-2 ${ }^{* \star}$ & AHCX01000000.1 & 93/96 & $100 / 100$ \\
\hline B. toyonensis Rock1-3* & CM000728.1.1 & $100 / 100$ & $100 / 100$ \\
\hline B. toyonensis BAG1O-2* & AHCO00000000.1 & $100 / 100$ & $100 / 100$ \\
\hline B. toyonensis HuB4-10** & AHEE00000000.1 & $100 / 100$ & $100 / 100$ \\
\hline B. toyonensis VD214** & AHFN00000000.1 & $100 / 100$ & 99/99 \\
\hline B. toyonensis HuB2-9** & AHED00000000.1 & $100 / 100$ & 99/99 \\
\hline B. toyonensis Rock3-29* & CM000731.1 & $100 / 100$ & 99/99 \\
\hline B. toyonensis Rock4-18** & CM000735.1.1 & 93/96 & 99/99 \\
\hline
\end{tabular}

${ }^{*}$ Clustered to the B. toyonensis group by Jiménez et al. (2013); ${ }^{* *}$ Clustered to the B. toyonensis group by Liu et al. (2015); ${ }^{* \star *}$ TS, truncated sequence detected. In some cases the NCBI Reference for master record for a whole genome shotgun sequence is given.

TABLE 3 | Breakdown of the genomic sequences analyzed to identify homologs of the B. toyonensis BCT-7112 ${ }^{\top}$ TetM and CatQ proteins.

\begin{tabular}{lcccc}
\hline Taxonomic group/ID & Complete & Chromosome & Scaffold & Contig \\
\hline B. anthracis/1392 & 44 & 10 & 41 & 42 \\
B. bombysepticus/1330043 & 1 & 0 & 0 & 0 \\
B. cereus (sensu stricto)/1396 & 32 & 29 & 154 & 51 \\
B. mycoides/1405 & 2 & 3 & 3 & 5 \\
B. thuringiensis/1428 & 37 & 0 & 14 & 12 \\
B. toyonensis/155322 & 1 & 0 & 0 & 0 \\
B. weihenstephanensis/86662 & 2 & 0 & 0 & 4 \\
B. wiedmannii/1890302 & 0 & 0 & 13 \\
\hline
\end{tabular}

TABLE 4 | The distribution of proteins encoded by homologs (>85\% similarity) of the tetM and catQ genes of $B$. toyonensis BCT-7112 $^{\top}$ among other members of the $B$. cereus sensu lato group.

\begin{tabular}{|c|c|c|c|c|c|c|c|c|c|}
\hline Gene & B. thuringiensis & $\begin{array}{c}\text { B. cereus } \\
\text { (sensu stricto) }\end{array}$ & B. anthracis & B. mycoides & $\begin{array}{c}\text { B. weihen- } \\
\text { stephanensis }\end{array}$ & B. toyonensis & B. wiedmannii & B. bombysepticus & Total \\
\hline CatQ & 66 & 161 & 111 & 7 & 6 & 18 & 11 & 1 & 381 \\
\hline
\end{tabular}

and have similar growth kinetics and therefore only the growth profiles of strain Rock1-3 are shown in Figure S3, in comparison with the profiles of strains BCT-7112 ${ }^{\mathrm{T}}$, BCT-7112 $\Delta$ tet, and BCT-7112 $\Delta$ cat.

When B. toyonensis BCT-7112 $2^{\mathrm{T}}$ was grown in the presence of various concentrations of tetracycline $(0,1,2,4,16$, and
$32 \mu \mathrm{g} / \mathrm{ml}$ ) the MIC was $16 \mu \mathrm{g} / \mathrm{ml}$ (Figure S3A). When the isogenic strain (BCT-7112 $\Delta$ tet) in which the tetM (Btoyo_0322) gene had been replaced with a spectinomycin resistance gene was tested, the MIC was $1 \mu \mathrm{g} / \mathrm{ml}$ (Figure S3B). In contrast, when the isogenic strain (BCT-7112 $\Delta$ cat) in which the catQ gene was replaced with a spectinomycin resistance gene, the MIC for 
tetracycline remained at $16 \mu \mathrm{g} / \mathrm{ml}$ (Figure S3C). In the case of the Rock1-3 strain, there was some initial growth at $1 \mu \mathrm{g} / \mathrm{ml}$ (Figure S3D), but the cells eventually died and the biomass concentration declined, indicating it had an MIC for tetracycline of $1 \mu \mathrm{g} / \mathrm{ml}$.

Similar growth experiments were performed to determine the MICs for chloramphenicol of $B$. toyonensis strains BCT-7112 ${ }^{\mathrm{T}}$, BCT-7112 $\Delta$ tet, BCT-7112 $\Delta$ cat, and Rock1-3 (Figures S3E-H). These confirmed that cat $Q$ was responsible for the chloramphenicol resistance phenotype and that the Rock1-3 strain, with an MIC of $1 \mu \mathrm{g} / \mathrm{ml}$, was susceptible to this antibiotic.

Previous studies indicated an involvement of the gerIC-nucB intergenic region of plasmid pBCT77 in contributing to, or enhancing, the observed resistance of B. toyonensis BCT-7112 to tetracycline and chloramphenicol (EFSA-FEEDAP, 2014; Matsumoto, Asahi Vet Japan, personal communication). We therefore confirmed the presence of the gerIC-nucB intergenic region in $B$. toyonensis strains BCT-7112 ${ }^{\mathrm{T}}$, BCT-7112 $\Delta$ tet, and BCT-7112 $\Delta$ cat and also generated versions of these strains with additional copies of this region on plasmid pIGR19 (Section Construction of Strains with Additional Copies of the gerIC-nucB Intergenic Region). In addition, a strain of B. toyonensis Rock1-3 was generated with additional copies of the intergenic region on pIGR1. The resulting strains were grown in the presence of various concentrations of tetracycline (Figures S4A-D) and chloramphenicol (Figures S4E-G) to determine the influence of the intergenic region on growth and resistance. The data (Figure S4) indicated that the presence of additional copies of the gerIC-nucB locus do not significantly influence the growth profiles of $B$. toyonensis strains BCT-7112 ${ }^{\mathrm{T}}$, BCT-7112 $\Delta$ tet, and BCT-7112 $\Delta$ cat on either of the antibiotics. In the case of strain Rock1-3, which encodes TetM and CatQ proteins that are identical to those in strain BCT-7112 ${ }^{\mathrm{T}}$, but which is susceptible to tetracycline and chloramphenicol, the presence of the gerIC-nucB intergenic region had no impact it's sensitivity to these antibiotics. Taken together, these data rule out any impact of the $\operatorname{ger} I C-n u c B$ intergenic region of pBCT77 as a factor in the resistances of $B$. toyonensis BCT- $7112^{\mathrm{T}}$ to these antibiotics.

\section{Why Are Strains Encoding TetM and CatQ Susceptible to Tetracycline and Chloramphenicol?}

B. toyonensis strains Rock1-3 and Rock3-28 encode identical or almost identical TetM and CatQ proteins to those encoded by strain BCT-7112 ${ }^{\mathrm{T}}$ (Table 2), but both are susceptible to tetracycline and chloramphenicol (MICs $1 \mu \mathrm{g} / \mathrm{ml}$ ). We observed that strains Rock1-3 and Rock3-28 tended to aggregate at or close to their MIC and therefore monitored their morphologies while growing in LB medium in the presence of $2 \mu \mathrm{g} / \mathrm{ml}$ of the antibiotics, comparing them with the morphologies of strain BCT-7112 ${ }^{\mathrm{T}}$ growing in the presence of the antibiotics with either 2 and $32 \mu \mathrm{g} / \mathrm{ml}$. Samples were taken every $60 \mathrm{~min}$ for microscopy and a representative set of data for growth in the presence of chloramphenicol is shown in Figure 2. During the first $60 \mathrm{~min}$ all of the cells appeared normal. However, from $\sim 120$ min the morphologies of the Rock1-3 and Rock328 strains exhibited distorted cell morphologies compared with BCT-7112 ${ }^{\mathrm{T}}$ (Figure 2). The observed aggregation of the Rock13 and Rock3-28 strains is therefore likely due to this disturbed morphology that ultimately results in cell death.

We next addressed the question of whether the tet $M$ and cat $Q$ genes in strains Rock1-3 and Rock3-28 were expressed. To do this we carried out both Northern blot and qPCR analyses. Northern blotting analysis was carried out on $B$. toyonensis strains BCT$7112^{\mathrm{T}}$, BCT-7112 $\Delta$ cat, BCT-7112 $\Delta$ tet, Rock1-3, and Rock3-28. Cultures were grown in LB to mid-exponential phase and total RNA extracted and electrophoresed on two identical denaturing ( $1 \%$ formalin) agarose gels; to increase sensitivity, $\sim 5$ times more RNA was added to the tracks of strains Rock1-3 and Rock3-28 compared with that of strain BCT-7112 ${ }^{\mathrm{T}}$. Following, hybridization with a DIG-labeled catQ probe, monocistronic catQ-specific transcripts $(\sim 0.8-\mathrm{kb})$ were detected in strains BCT$7112^{\mathrm{T}}$ and BCT-7112 $\Delta$ tet, but not the other strains (Figure 3A). In the case of the tet $M$-specific probe, strong monocistronic tet $M$ specific transcripts $(\sim 2.0-\mathrm{kb})$ were detected in strains BCT-7112 ${ }^{\mathrm{T}}$ and BCT-7112 $\Delta$ cat, and weak transcripts in strains Rock1-3 and Rock3-28 (Figure 3B). As expected a catQ-specific transcript was absent from strain BCT-7112 $\Delta$ cat and a tetM-specific transcript from strain BCT-7112 $\Delta$ tet.

The Northern blot expression data were confirmed by qPCR using $g y r B$ as the baseline control. B. cereus ATCC 14579 and $B$. thuringiensis ATCC 10792 were included as both encode homologs of the TetM, while B. thuringiensis ATCC 10792 only encodes a copy of CatQ. The qPCR data (Table 5) show expression of tet $M$ in BCT-7112 ${ }^{\mathrm{T}}$ and BCT-7112 $\Delta$ cat, but considerably lower or no expression in Rock1-3, BCT-7112 $\Delta$ tet, ATCC 14579, and ATCC 10792. In the case of the catQ qRNA data, expression was detected in BCT-7112 ${ }^{\mathrm{T}}$, BCT-7112 $\Delta$ tet, but again, little or no expression of this gene in BCT-7112 $\Delta$ cat, Rock1-3, ATCC 14579, and ATCC 10792 (Table 5).

Since B. toyonensis strains Rock1-3 and Rock3-28 each contained homologs of the BCT-7112 ${ }^{\mathrm{T}}$ TetM and CatQ proteins with a high level of identity (Table 2), we carried out experiments to determine if their cognate genes were expressed in the related bacterium B. subtilis strain 168. The strategy involved cloning their coding sequences, together with upstream regulatory sequences, into the $B$. subtilis integration/expression vector pDR111. The genes and upstream sequences from B. toyonensis BCT-7112 ${ }^{\mathrm{T}}$ were also cloned as a control. In each case the tet $M$ and catQ sequences were cloned downstream of the IPTG inducible $\mathrm{P}_{\text {hyper-spank }}$ promoter in $\mathrm{pDR} 111$ and the resulting expression cassette was integrated at the non-essential amyE locus of $B$. subtilis. The resulting constructs allow for the expression of the cognate genes from their native promoters or, following IPTG induction, from the $\mathrm{P}_{\text {hyper-spank }}$ promoter. The growth profiles of the $B$. subtilis strains grown in LB medium with various concentrations of tetracycline or chloramphenicol $(0,4,8,16$, and $32 \mu \mathrm{g} / \mathrm{ml})$ and with or without IPTG are shown in Figure 4.

B. subtilis is unable to grow in the presence of $4 \mu \mathrm{g} / \mathrm{ml}$ of tetracycline (Figure 4A). Cloning the tet $M$ gene from $B$. toyonensis BCT-7112 ${ }^{\mathrm{T}}$ into B. subtilis 168 allows this strain to 


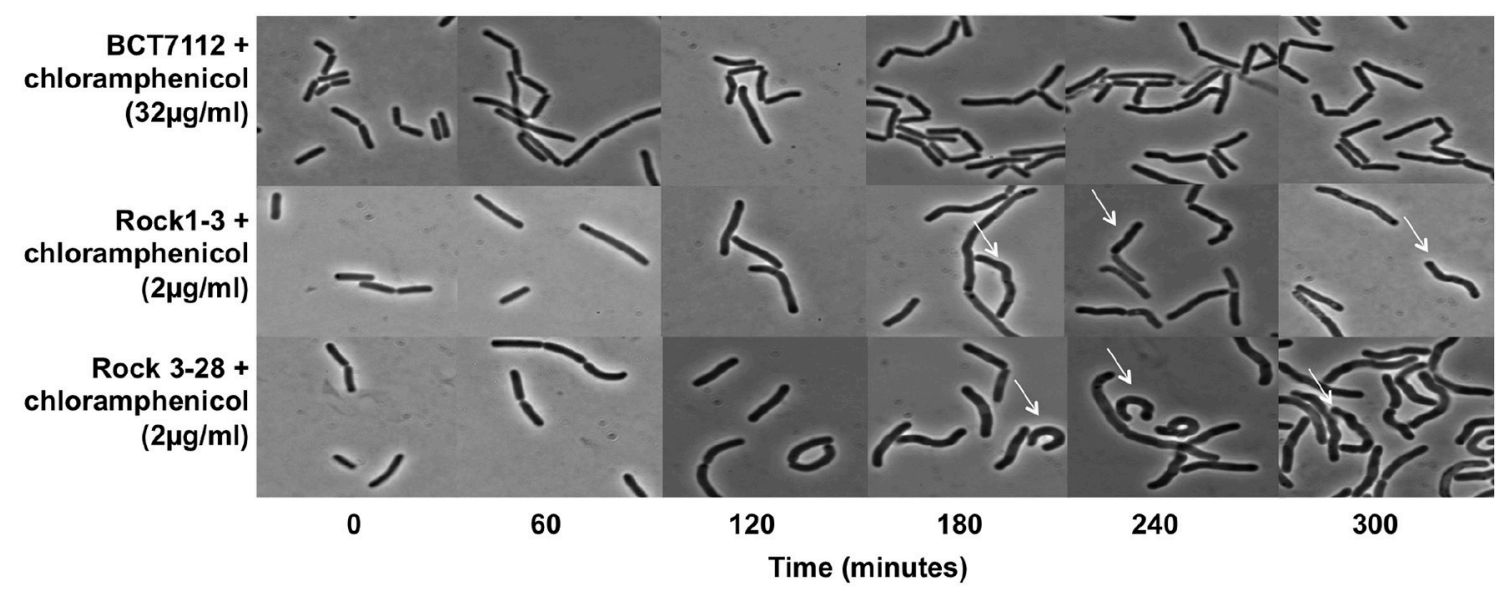

FIGURE 2 | Influence of chloramphenicol on cell morphology. B. toyonensis strains BCT-7112 ${ }^{\top}$, Rock1-3, and Rock3-28 were grown in LB medium at concentrations of chloramphenicol just above their MICs. Samples were taken every 60 min and viewed by phase-contrast microscopy. In the case of the Rock $1-3$ and Rock3-28, twisted cells can be seen in the samples taken at 180 min and later (white arrows).

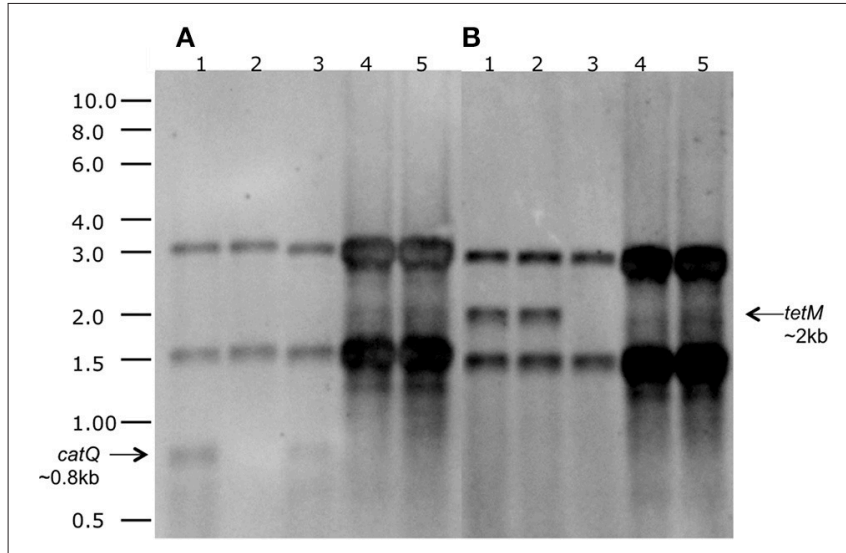

FIGURE 3 | Northern blot analysis for the detection of catQ and tetM transcripts in strains of $\boldsymbol{B}$. toyonensis. (A) catQ-specific probe; (B) tetM-specific probe. The tracks contained total RNA from the following strains: 1. ВCT-7112 ${ }^{\top}$; 2. ВCT-7112 $\Delta$ cat; 2. BCT-7112 $\Delta$ tet; 4. Rock1-3; 5.

Rock3-28. Approximately 5 times more RNA was added to the tracks containing Rock1-3 and Rock3-28 to improve sensitivity. The two prominent bands present in all the tracks are the 30S rRNA (upper) and 16S rRNA (lower).

grow in the presence of $32 \mu \mathrm{g} / \mathrm{ml}$, albeit with an extended lag phase (Figure 4B). The addition of IPTG to the culture medium did not have a significant influence of growth up to $32 \mu \mathrm{g} / \mathrm{ml}$ tetracycline, indicating that the upstream regulatory region of tet $M$ was fully functional in this bacterium (Figure 4B). The growth kinetics for the B. subtilis strains encoding the tetM genes from strains Rock1-3 (Figures 4C,D) and Rock3-28 (Figures 4F,G) were similar and are discussed together. The data show that, in the absence of IPTG, the B. subtilis strains encoding the tet $M$ genes from these strains grew in the presence of 32 $\mu \mathrm{g} / \mathrm{ml}$ tetracycline. However, the growth kinetics show that the lag phase increased in length with increasing concentrations of tetracycline, to the extent that growth at $32 \mu \mathrm{g} / \mathrm{ml}$ tetracycline
TABLE 5 | Quantitative PCR analysis of tetM and catQ transcripts in B. toyonensis strains $B C T-7112^{\top}$, BCT-7112 $\Delta$ cat, BCT-7112 $\Delta$ tet, and Rock1-3, B. cereus ATCC 14579 and B. thuringiensis ATCC 10792.

\begin{tabular}{|c|c|c|}
\hline Transcript & Strain & $R=2^{\Delta c t}$ \\
\hline \multicolumn{3}{|l|}{ tetM } \\
\hline & B. toyonensis BCT-7112 ${ }^{\top}$ & 9.80 \\
\hline & B. toyonensis BCT-7112 $\Delta$ cat & 5.91 \\
\hline & B. toyonensis BCT-7112 $\Delta$ tet & 0.0 \\
\hline & B. toyonensis Rock1-3 & 0.18 \\
\hline & B. cereus ATCC 14579 & 0.59 \\
\hline & B. thuringiensis ATCC 10792 & 0.0 \\
\hline \multicolumn{3}{|l|}{ catQ } \\
\hline & B. toyonensis BCT-7112 ${ }^{\top}$ & 233.95 \\
\hline & B. toyonensis BCT-7112 $\Delta \mathrm{cat}$ & 0.13 \\
\hline & B. toyonensis BCT-7112 $\Delta$ tet & 1706.9 \\
\hline & B. toyonensis Rock1-3 & 3.56 \\
\hline & B. cereus ATCC 14579 & 0.71 \\
\hline & B. thuringiensis ATCC 10792 & 0.01 \\
\hline
\end{tabular}

is only observed after approximately $18 \mathrm{~h}$. When IPTG is added, the cultures grew without a lag phase up to $8 \mu \mathrm{g} / \mathrm{ml}$ tetracycline and with considerably shorter lag phases at 16 and $32 \mu \mathrm{g} / \mathrm{ml}$ tetracycline. Taken together, the data confirm that the tet $M$ genes in B. toyonensis strains Rock1-3 and Rock3-28 encode functional proteins, but that expression from their native promoters is weak.

Similar results were observed when $B$. subtilis encodes the catQ genes from B. toyonensis strains BCT-7112 ${ }^{\mathrm{T}}$, Rock1-3, and Rock3-28 following growth on chloramphenicol $(0,4,8,16$, and $32 \mu \mathrm{g} / \mathrm{ml}$ ). While $B$. subtilis alone is unable to grow in the presence of $4 \mu \mathrm{g} / \mathrm{ml}$ of chloramphenicol (Figure $4 \mathbf{H}$ ), the inclusion of the catQ gene from strain BCT-7112 ${ }^{\mathrm{T}}$, together with its native promoter, facilitated growth up to $16 \mu \mathrm{g} / \mathrm{ml}$ (Figure $4 \mathrm{I}$ ). However, with increasing concentrations of chloramphenicol, 


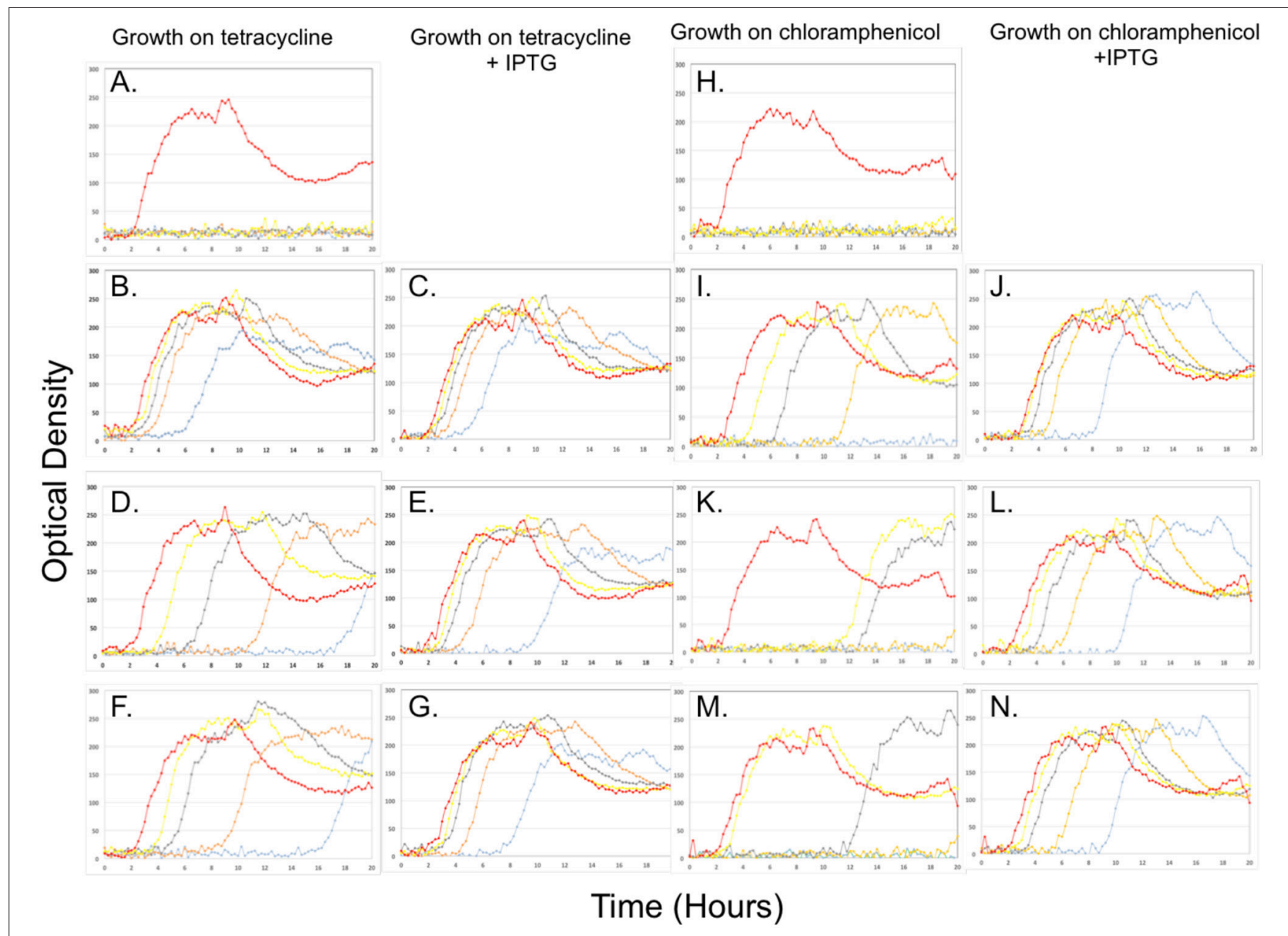

FIGURE 4 | Growth of $B$. subtilis strain 168 (A,H), with tetM (A-G) or catQ (H-N) genes from BCT-7112 ${ }^{\top}$ (B,C,I,J), Rock1-3 (D,E,K,L) and Rock3-28 (F,G,M,N) on various concentrations of tetracycline $\mathbf{( A - G )}$ or chloramphenicol $\mathbf{( H - N )}$ with $\mathbf{( C , E , G , J , L , N )}$ or without $(\mathbf{A}, \mathbf{B}, \mathbf{D}, \mathbf{F}, \mathbf{H}, \mathbf{I}, \mathbf{K}, \mathbf{M})$ the addition of IPTG (1 mM). The cultures were grown in a BioLector microreactor that determines optical density by measuring the scattered light signal (see Section Visualization of Cells by Light Microscopy). The data is shown on a linear rather than logarithmic graph to make it easier to compare growth profiles. The antibiotic concentrations used were ( $\mu \mathrm{g} / \mathrm{ml}): 0, \mathrm{red} ; 4$, yellow; 8 , gray; 16 , orange and 32 , light blue).

there was a longer lag before the culture started to grow, indicating that the native cat $Q$ promoter was relatively efficient in B. subtilis. This was confirmed when IPTG was added to the culture; the lag phases were considerably reduced and the culture grew at $32 \mu \mathrm{g} / \mathrm{ml}$ (Figure $4 \mathrm{~J}$ ). The growth kinetics for the $B$. subtilis strains encoding the cat $Q$ genes from $B$. toyonensis strains Rock1-3 (Figures 4K,L) and Rock3-28 (Figures 4M,N) were similar with their native promoters facilitating growth at concentrations up to $8 \mu \mathrm{g} / \mathrm{ml}$, albeit with lag phases different length (Figures 4K,M). When IPTG was included in the culture medium, the cells were able to grow in the presence of $32 \mu \mathrm{g} / \mathrm{ml}$, but with longer lag phases at the higher antibiotic concentrations (Figures 4L,N).

Taken together, the data in Figure $\mathbf{4}$ clearly show that the tet $M$ and cat $Q$ genes of B. toyonensis strains Rock1-3 and Rock328 encode function proteins, capable of conferring resistance to their cognate antibiotics. The data also suggests an explanation for why B. toyonensis strain BCT-7112 ${ }^{\mathrm{T}}$ exhibits resistance to these antibiotics, while strains Rock1-3 and Rock3-28 do not (Figure S3), namely that their upstream promoters are relatively inefficient. Alignment of the nucleotides upstream of tet $M$ and catQ coding sequences indicates a high degree of identity between the regulatory regions of these genes from strains BCT-7112 $2^{\mathrm{T}}$, Rock1-3, and Rock3-28 (Figure 5). Figure 5 also identifies consensus sequences for putative Sigma A (vegetative) promoters and single nucleotide polymorphisms (SNPs) with respect to BCT-7112 $2^{\mathrm{T}}$. In the case of tet $M$, identical SNPs occur within the putative -35 sequences and within the sequences between the -35 and -10 sequences in Rock1-3 and Rock3-28, with the latter having three additional SNPs in this region (Figure 5A). In the region upstream of cat $Q$, three putative Sigma A promoters were identified, the most peripheral of which contains the only SNP observed in this region (Figure 5B). 


\begin{tabular}{|c|c|}
\hline & \\
\hline & $\begin{array}{ll}-35 \\
\end{array}$ \\
\hline ВСТ7112 & GCAAGCATTTTGTTTTCATTCTATTTTTTCCAAAATAAGGCGCTTTGTAGCTTTACAAAA \\
\hline & GCAAGCATTTTGTTTTCATTCTATTTTTTCCAAAATAAGGCGСTTTGTAGCGTTACAAAA \\
\hline & $\begin{array}{c}\text { GCAAGCATTTTTTTTCATTCTATTTTTTCCAAATTAAGGCGCTTTGTAGCGITACAAAA } \\
-10\end{array}$ \\
\hline ВСТ7112 & AATGCAAATTACATTATAATAGTAATAATTTAAATGACTGGAGTGTGATTGTAATGCGGC \\
\hline Roc] & АATGTAAATTACATTATAATAGTAATAATTTAAATGACTGGAGTGTGATTGTAATGCGGC \\
\hline RoC & $\begin{array}{c}\text { AATGTAAATTACATTATAATAGTAATAATTTAAATGACTGGAGAGTGATTGTAATGCGGC } \\
\text { RBS }\end{array}$ \\
\hline ВСТ & AATACACAAATAGAAATGAGGAAATGAAAATGGAAAATGAAATGAGGATATTTATATATG \\
\hline Roc & AATACACAAATAGAAATGAGGAAATGAAAATGGAAAATGAAATGAGG \\
\hline Rock3-28 & AATACACAAATAGAAATGAGGAAATGAAAATGGAAAATGAAATGAGGATATTCACATATG \\
\hline в catQ & \\
\hline & $\begin{array}{lll}-35 & -35 \\
\end{array}$ \\
\hline $\mathrm{BCT}$ & САСАTTATTAATAGTATGTGGATGGATTGCAAGAAATATGTGGATGGATTTTTGCAAATG \\
\hline Roc] & САСАTTATTAATAGTATGTGGATGGATTGCAAGAAATATGTGAATGGATTTTTGCAAATG \\
\hline Rock3-28 & $\begin{array}{ccc}\text { CACATTATTAATAGTATGTGGATGGATTGCAAGAAATATGTGAATGGATTTTTGCAAATG } \\
-10 \quad-10\end{array}$ \\
\hline BCT & TTATATAATTAAATAAAAACGTGAGTATAAAT \\
\hline Rock & TTATATAATTAAATAAAAACGTGAGTATAAATC \\
\hline Rock3-28 & $\begin{array}{ccc}\text { TTATATAATTAAATAAAAACGTGAGTATAAATGTAAACTATAAGGAGTGAACAAGTGTGG } \\
-35 & -10 & \text { RBS }\end{array}$ \\
\hline BCT & TGAAAACAAGGAAATTAGCCTTCTAAAATAAAAATGAAATTTTAGGAGGCTAATGAAATG \\
\hline Roc & IGAAAACAAGGAAATTAGCСTTCTAAAATAAAAATGAAATTTTAGGAGGCTAATGAAATG \\
\hline Rock3-28 & TGAAAACAAGGAAATTAGCCTTCTAAAATAAAAATGAAATTTTAGGAGGCTAATGAAATG \\
\hline
\end{tabular}

FIGURE 5 | Nucleotide sequences upstream of the (A) tetM and (B) catQ genes of B. toyonensis strains BCT-7112 ${ }^{\top}$, Rock1-3, and Rock3-28. Putative Sigma A (vegetative) promoter consensus sequences are shown highlighted $(-35 /-10)$ and the ribosome binding sites (RBS) and start codons are in bold. Single nucleotide polymorphisms (SNPs) in the Rock1-3 and Rock3-28 strains are also highlighted.

\section{DISCUSSION}

B. toyonensis BCT-7112 ${ }^{\mathrm{T}}$ is resistant to tetracycline and chloramphenicol and the annotated genome and plasmid sequences were used to identify the putative genes involved. Reciprocal recombination was then used to replace the target resistance genes with a spectinomycin resistance gene and this unambiguously identified Btoyo_0332 (tetM) and Btoyo_4985 $($ cat $Q)$ as being the genes responsible for the resistance phenotypes in B. toyonensis BCT-7112 ${ }^{\mathrm{T}}$.

We addressed the question of whether the genes responsible for tetracycline and chloramphenicol resistances in BCT-7112 ${ }^{\mathrm{T}}$ were intrinsic or acquire in three ways. Firstly we established that these genes were not associated with known MGEs (e.g., transposon, transposase, insertion sequence, conjugation etc.). Secondly, we analyzed their \%GC content in relation to the genome as a whole and showed that they were not significantly different. Thirdly, we showed that homologous genes were not only present in closely related species, but were also present in virtually all representative of the $B$. cereus group sensu lato.
The data (Tables 2, 4) clearly show that the tet $M$ and cat $Q$ genes are widely distributed among members of the $B$. cereus group sensu lato, are located within the same genomic neighborhoods and are therefore unambiguously of ancient origin within this group, rather than having been acquired as the result of more recent horizontal gene transfer. This conclusion is supported by animal feeding experiments in which $\mathrm{qPCR}$ was used to quantify tetracycline and chloramphenicol resistance genes in intestinal samples from piglets and cattle (CasanovasMassana et al., 2014). The conclusion from these studies is that the presence of $B$. toyonensis does not contribute significantly to the antibiotic resistance load already present in the intestinal tract of these animals.

While B. toyonensis BCT- $7112^{\mathrm{T}}$ is resistant to tetracycline and chloramphenicol, closely related strains (e.g., Rock1-3 and Rock3-28) with identical or virtually identical genes and upstream regulatory regions are susceptible to these antibiotics. To address this apparent contradiction, we sought answers to two specific questions: are the cognate resistance genes in Rock1-3 and Rock3-28 functional and, if so, why do they not elaborate the expected resistance phenotype? Using Rock1-3 and Rock3-28 
as representative strains, we integrated their tet $M$ and cat $Q$ genes, together with their upstream regulatory sequences, into the $a m y E$ locus of B. subtilis. The data (Figure 4) show firstly that the products of these genes are functionally active since induction of an upstream $\mathrm{P}_{\text {hyper-spank promoter conferred the cognate }}$ resistance on the $B$. subtilis host. More significantly, when using their native regulatory sequences, these genes were able to confer resistance to $B$. subtilis at levels above its $\mathrm{MIC}(\sim 1 \mu \mathrm{g} / \mathrm{ml})$, albeit with varying degrees of efficiency and extended lag phases. This suggests that the native promoters of these genes are relatively weak, and this was confirmed by analysis of their transcript levels (Figure 3 and Table 5). Analysis of cells taken from the non-induced cultures of $B$. toyonensis Rock1-3 growing on tetracycline $(16 \mu \mathrm{g} / \mathrm{ml}$, Figure 4D) and chloramphenicol ( $8 \mu \mathrm{g} / \mathrm{ml}$, Figure $4 \mathrm{~K}$ ) revealed that the majority of the cells had become resistant to the cognate antibiotic, presumably by the selection of cells within the population with spontaneous mutations in either the native upstream regulatory sequences or elements of the $\mathrm{P}_{\text {hyper-spank }}$ promoter system. It is likely that the extended lag phases in these cultures reflect the time taken for such mutants to accumulate in the population.

The final question we addressed is why the $B$. cereus sensu lato group encodes genes that are so poorly expressed that they do not register a phenotype in the standard protocols used for regulatory purposes to identify MIC values. Although, we cannot provide a definitive answer this question, their maintenance over such a long evolutionary time-scale suggests that they must have a specific role or selective advantage in their natural environment, the soil. One possibility is that they are transcribed from nonvegetative Sigma factor promoters induced, for example, in response to specific stresses, quorum sensing pheromones or differentiation processes, and that are not encountered under the MIC test conditions. Another is that their expression has been down regulated over evolutionary time by single base-pair mutations that, as a bet-hedging strategy (Ferenci and Maharjan, 2015), are able to revert at a high frequency and subsequently selected under appropriate conditions. This might account for the resistance phenotypes of B. toyonensis BCT-7112 ${ }^{\mathrm{T}}$.

The results represent a potential issue for regulatory authorities who tend to rely on phenotypic data for assessing the implications of introducing antibiotic resistance organisms into the food chain. For example, in their conclusions on the antibiotic susceptibility of B. toyonensis NCIMB $14858^{\mathrm{T}}$ (i.e., BCT-7112T) the EFSA FEEDAP Panel stated the following: "Although, there is some evidence of the presence of the cat $Q$ and tet $(M)$ genes in eight closely related strains, none of these demonstrated the resistant phenotype. Consequently, resistance to chloramphenicol and tetracycline cannot be considered intrinsic to the newly defined species" (EFSA-FEEDAP, 2014). This statement raises the important issue of exactly what evidence regulatory authorities should consider before concluding whether or not a resistance factor (genotype or phenotype) is intrinsic. The traditional reliance on phenotype is clearly insufficient in the post-genomic era since the intrinsic nature of an observed phenotype can only be defined at the level of its gene. This is particularly the case with resistance determinants since the same phenotype can be encoded by entirely different mechanisms (Blair et al., 2015). Moreover, detecting homologous genes in both closely related and distantly related organisms, particularly if located at similar genomic neighborhoods, strengthens the case for recognizing that the gene, rather than it observable phenotype, is intrinsic.

The question remains as to whether or not strains that are intrinsically resistant to antibiotic(s) should be used in any process associated with the human food chain? It is likely that antibiotics (also referred to as secondary metabolites) have been produced for over 500 million years, dating back to the Cambrian period (Baltz, 2008; Cox and Wright, 2013). Their production relates to evolutionary processes taking place millions of years before the use of antimicrobial chemotherapy, when the antibiotics encountered by bacteria were produced by competitor organisms in the same environment (Perry and Wright, 2013). In some cases, the evolution of impermeability barriers such as the outer membrane, conferred resistance (e.g., erythromycin resistance in Escherichia coli), in others existing genes were duplicated and modified (e.g., the elongation factor TU in the case if TetM) or were acquired from the producers themselves (e.g., MFS efflux pumps). It would be unrealistic to exclude the use of all such strains. A realistic approach requires a detailed analysis that: (i) identifies the gene(s) responsible for the resistance phenotype, (ii), confirms that they are not associated with known MGEs (e.g., plasmids, prophages, transposons, insertion sequence, resistance cassettes, integrons etc.), and (iii) irrespective of phenotype, that homologous genes are present in related strains/species and at similar genomic locations.

In conclusion our data clearly show that the tet $M$ and catQ genes of B. toyonensis BCT-7112 ${ }^{\mathrm{T}}$ are intrinsic not only to this species, but also to the $B$. cereus group (sensu lato). They are confirmed to be of ancient origin, since genes subject to horizontal gene transfer would be associated with MGEs, have a significantly different \%GC and located at a variety of genomic neighborhoods. The proven intrinsic nature and nontransferability of these antibiotic resistance genes in B. toyonensis BCT- $7112^{\mathrm{T}}$ counts in favor of recommending the safe use of this strain as an additive in animal nutrition. These studies also show that intrinsic resistance should be defined at the genomic rather than the phenotypic level and this should be taken into account whenever a scientific assessment of the nature and genetic basis of a bacterial antibiotic resistance is performed.

\section{AUTHOR CONTRIBUTIONS}

All authors were involved in the design of the studies and in the interpretation of the data. HG and SP carried out the majority of the experimental work at Newcastle University, under the supervision of $\mathrm{CH}$. FN and EM carried out the qPCR experiments at the Hospital de la Santa Creu i Sant Pau. In addition to their involvement in the design of the studies, $G J$ and $A B$ provided background information, strains and a critical input into the preparation of the manuscript.

\section{ACKNOWLEDGMENTS}

We are grateful to the US Naval Medical Research Center, Rockville, for the supply of B. toyonensis (previously, B. cereus) strains Rock1-3 and Rock3-28. This work was carried out 
independently in laboratories at Newcastle University (UK) and the Hospital de la Santa Creu i Sant Pau (Spain).

\section{SUPPLEMENTARY MATERIAL}

The Supplementary Material for this article can be found online at: http://journal.frontiersin.org/article/10.3389/fmicb. 2016.02122/full\#supplementary-material

Table S1 | BLASTp was used to identify homologs of the $B$. toyonensis $7^{7112}{ }^{\top}$ TetM protein against sequences in the NCBI genome database (Altschul et al., 1990). The data was downloaded in CVS format and uploaded into Excel. The data shows the Reference Sequence IDs, individual Sequence IDs, the description of the protein in the annotation file, the bacterial source, alignment score, $E$-value and \% identity.

\section{Table S2 | BLASTp was used to identify homologs of the B. toyonensis} $7112^{\top}$ CatQ protein against sequences in the NCBI genome database (Altschul et al., 1990). The data was downloaded in CVS format and uploaded into Excel. The data shows the Reference Sequence IDs, individual Sequence IDs, the description of the protein in the annotation file, the bacterial source, alignment score, $E$-value and \% identity.

Figure S1 | Neighbor Joining tree of homologs of the B. toyonensis BCT-7112 ${ }^{T}$ TetM tetracycline resistance protein (Btoyo_0322) identified in the BLASTp analysis. The algorithm used produces an un-rooted tree (Saitou and Nei, 1987). The maximum allowed fraction of mismatched bases between any pair of sequences was 0.85 . The evolutionary distance between two sequences

\section{REFERENCES}

Altschul, S. F., Gish, W., Miller, W., Myers, E. W., and Lipman, D. J. (1990). Basic local alignment search tool. J. Mol. Biol. 215, 403-410. doi: 10.1016/S0022-2836(05)80360-2

Anagnostopoulos, C., and Spizizen, J. (1961). Requirement for transformation in Bacillus subtilis. J. Bacteriol. 81, 741-746.

Baltz, R. H. (2008). Renaissance in antibacterial discovery from actinomycetes. Curr. Opin. Pharmacol. 8, 557-563. doi: 10.1016/j.coph.2008.04.008

Bavykin, S. G., Lysov, Y. P., Zakhariev, V., Kelly, J. J., Jackman, J., Stahl, D., et al. (2004). Use of $16 \mathrm{~S}$ rRNA, 23S rRNA, and gyrB gene sequence analysis to determine phylogenetic relationships of Bacillus cereus group microorganisms. J. Clin. Microbiol. 42, 3711-3730. doi: 10.1128/JCM.42.8.3711-3730.2004

Beck, C. F., Mutzel, R., Barbé, J., and Müller, W. (1982). A multifunctional gene (tetR) controls Tn10-encoded tetracycline resistance. J. Bacteriol. 150, 633-642.

Blair, J. M., Webber, M. A., Baylay, A. J., Ogbolu, D. O., and Piddick, L. J. V. (2015). Molecular mechanisms of antibiotic resistance. Nat. Rev. Microbiol. 13, 42-51. doi: $10.1038 /$ nrmicro3380

Blanch, A. R., Méndez, J., Castel, S., and Reina, M. (2014). Comparison of procedures for the extraction of supernatants and cytotoxicity tests in Vero cells, applied to asses the toxigenic potential of Bacillus spp. and Lactobacillus spp., intended for use as probiotic strains. J. Microbiol. Methods 103, 64-69. doi: 10.1016/j.mimet.2014.05.019

Böhm, M.-E., Huptas, C., Krey, V. M., and Schere, S. (2015). Massive horizontal gene transfer, strictly vertical inheritance and ancient duplications differentially shape the evolution of Bacillus cereus enterotoxin operons hbl, cytK and nhe. BMC Evol. Biol. 15:246. doi: 10.1186/s12862-015-0529-4

Britton, R. A., Eichenberger, P., Gonzalez-Pastor, J. E., Fawcett, P., Monson, R., Losick, R., et al. (2002). Genome-wide analysis of the stationary-phase sigma factor (sigma-H) regulon of Bacillus subtilis. J. Bacteriol. 184, 4881-4890. doi: 10.1128/JB.184.17.4881-4890.2002

Casanovas-Massana, A., Sala-Comorera, L., and Blanch, A. R. (2014). Quantification of tetracycline and chloramphenicol resistance in digestive tracts of bulls and piglets fed with Toyocerin ${ }^{\circledR}$, a feed additive containing Bacillus toyonensis spores. Vet. Microbiol. 173, 59-65. doi: 10.1016/j.vetmic.2014.07.005 was modeled as the expected fraction of amino acid substitutions per site, based on the fraction of mismatched amino acids in the aligned region (Grishin, 1995).

Figure S2 | Neighbor Joining tree of the homologs of the B. toyonensis BCT-7112 ${ }^{\top}$ CatQ chloramphenicol resistance protein (Btoyo_4985) identified in the BLASTp analysis. The algorithm used produces an un-rooted tree (Saitou and Nei, 1987). The maximum allowed fraction of mismatched bases between any pair of sequences was 0.85 . The evolutionary distance between two sequences was modeled as the expected fraction of amino acid substitutions per site, based on the fraction of mismatched amino acids in the aligned region (Grishin, 1995).

Figure S3 | Minimum inhibitory concentrations (MICs) of strains of B. toyonensis on tetracycline (A-D) and chloramphenicol (E-H). (A/E), BCT-7112 $^{\top}$; (B/F), BCT-7112 $\Delta$ tet; (C/G), BCT-7112 $\Delta$ cat; (D/H) Rock1-3. The antibiotic concentrations used were $(\mu \mathrm{g} / \mathrm{mll})$ : 0 , red; 1 , green; 2 , dark blue; 4 , yellow; 8, gray; 16 , orange and 32 , light blue. The MIC values are shown for each set of growth curves. The experiments were carried out a minimum of three times and representative data show on a linear rather than logarithmic graph to make it easier to compare growth profiles.

Figure S4 | The impact of the gerIC-nucB intergenic region of plasmid pBCT77 on the tetracycline (A-D) and chloramphenicol (E-H) resistance profiles of the following strains: (A/E), BCT-7112(plGR1); (B/F),

BCT-7112 $\Delta$ tet(plGR1); (C/G), BCT-7112 $\Delta$ cat(plGR1); (D/H), Rock1-3(plGR1). The antibiotic concentrations used were ( $\mu \mathrm{g} / \mathrm{ml})$ : 0 , red; 1 , green; 2 , dark blue; 4 , yellow; 8, gray; 16 , orange and 32 , light blue. The MIC values are shown for each set of growth curves. The experiments were carried out a minimum of three times and representative data show on a linear rather than logarithmic graph to make it easier to compare growth profiles.

Chen, Y., Tenover, F. C., and Koehler, T. M. (2004). $\beta$-lactamase gene expression in a penicillin-resistant Bacillus anthracis strain. Antimicrob. Agents Chemother. 48, 4873-4877. doi: 10.1128/AAC.48.12.4873-4877.2004

Cox, G., and Wright, G. D. (2013). Intrinsic antibiotic resistance: mechanisms, origins, challenges and solutions. Int. J. Med. Microbiol. 303, 287-292. doi: 10.1016/j.ijmm.2013.02.009

Dönhöfer, A., Franckenberg, S., Wickles, S., Berninghausen, O., Beckmann, R., and Wilson, D. N. (2012). Structural basis for TetM-mediated tetracycline resistance. Proc. Natl. Acad. Sci. U.S.A. 109, 16900-16905. doi: $10.1073 /$ pnas. 1208037109

EFSA-FEEDAP (2012). Guidance on the assessment of bacterial susceptibility to antimicrobials of human and veterinary importance. EFSA J. 10:2740. doi: 10.2903/j.efsa.2012.2740

EFSA-FEEDAP (2014). Scientific Opinion on the safety and efficacy of Toyocerin ${ }^{\circledR}$ (Bacillus toyonensis) as a feed additive for chickens for fattening, weaned piglets, pigs for fattening, sows for reproduction, cattle for fattening and calves for rearing and for rabbits for fattening. EFSA J. 12:3766. doi: 10.2903/j.efsa.2014.3766

Ferenci, T., and Maharjan, R. (2015). Mutational heterogeneity: a key ingredient of bet-hedging and evolutionary divergence? Bioessays 37, 123-130. doi: 10.1002/bies.201400153

Grishin, N. V. (1995). Estimation of the number of amino acid substitutions per site when the substitution rate varies among sites. J. Mol. Evol. 42, 675-679. doi: $10.1007 / \mathrm{BF} 00175826$

Guinebretière, M.-H., Auger, S., Galleron, N., Contzen, M., De Sarrau, B., DeBuyser, M.-L., et al. (2013). Bacillus cytotoxicus sp. nov. is a new thermotolerant species of the Bacillus cereus group occasionally associated with food poisoning. Int. J. Syst. Evol. Microbiol. 63, 31-40. doi: 10.1099/ijs.0.03 0627-0

Homuth, G., Masuda, S., Mogk, A., Kobayashi, Y., and Schumann, W. (1997). The dnaK operon of Bacillus subtilis is heptacistronic. J. Bacteriol. 179, 1153-1164. doi: 10.1128/jb.179.4.1153-1164.1997

Jiménez, G., Urdiain, M., Cifuentes, A., López-López, A., Blanch, A. R., Tamames, J., et al. (2013). Description of Bacillus toyonensis sp. nov., a novel species of the Bacillus cereus group, and pairwise genome comparisons of the species of the group by means of ANI calculations. Syst. Appl. Microbiol. 36, 383-391. doi: 10.1016/j.syapm.2013.04.008 
Jung, M., Paek, W. K., Park, I., Han, J., Sin, Y., Paek, J., et al. (2010). Bacillus gaemokensis sp. nov., isolated from foreshore tidal flat sediment from the Yellow Sea. J. Microbiol. 48, 867-871. doi: 10.1007/s12275-010-0148-0

Jung, M. Y., Kim, J. S., Paek, W. K., Lim, J., Lee, H., Kim, P. I., et al. (2011). Bacillus manliponensis sp. nov., a new member of the B. cereus group isolated from foreshore tidal flat sediment. J. Microbiol. 49, 1027-1032. doi: 10.1007/s12275-011-1049-6

Kahlmeter, G., Brown, D. F., Goldstein, F. W., MacGowan, A. P., Mouton, J. W., Odenholt, I., et al. (2006). European Committee on Antimicrobial Susceptibility Testing (EUCAST) technical notes on antimicrobial susceptibility testing. Clin. Microbiol. Infect. 12, 501-503. doi: 10.1111/j.1469-0691.2006. 01454.x

Koehler, T. M., Dai, Z., and Kaufman-Yarbray, M. (1994). Regulation of the Bacillus anthracis protective antigen gene: $\mathrm{CO}_{2}$ and a trans-acting element activate transcription from one of two promoters. J. Bacteriol. 176, 586-595. doi: $10.1128 / \mathrm{jb} .176 .3 .586-595.1994$

Lechner, S., Mayr, R., Francis, K. P., Prüss, B. M., Kaplan, T., Wiessner-Gunkel, E., et al. (1998). Bacillus weihenstephanensis sp. nov. is a new psychrotolerant species of the Bacillus cereus group. Int. J. Syst. Bacteriol. 48, 1373-1381. doi: 10.1099/00207713-48-4-1373

Liu, B., Liu, G., Hu, G., Cetin, S., Lin, N., Tang, J., et al. (2014). Bacillus bingmayongensis sp. nov., isolated from the pit soil of Emperor Qin's Terra-cotta warriors in China. Antonie Van Leeuwenhoek 105, 501-510. doi: 10.1007/s10482-013-0102-3

Liu, Y., Lai, Q., Göker, M., Meier-Kolthoff, J. P., Wang, M., Sun, Y., et al. (2015). Genomic insights into the taxonomic status of the Bacillus cereus group. Sci. Rep. 5:14082. doi: 10.1038/srep14082

Oren, A., and Garrity, G. M. (2014). Validation list No. 155, List of new names and new combinations previously effectively, but not validly, published. Int. J. Syst. Evol. Microbiol. 64, 1-5. doi: 10.1099/ijs.0.060285-0

Perry, J. A., and Wright, G. D. (2013). The antibiotic resistance "mobilome": searching for the link between environment and clinic. Front. Microbiol. 4:138. doi: $10.3389 /$ fmicb. 2013.00138

Pfaffl, M. W. (2001). A new mathematical model for relative quantification in real-time RT-PCR. Nucleic Acids Res. 29, 2002-2007. doi: 10.1093/nar/ 29.9.e45

Rasko, D. A., Altherr, M. R., Han, C. S., and Ravel, J. (2004). Genomics of the Bacillus cereus group of organisms. FEMS Microbiol. Rev. 29, 303-329. doi: 10.1016/j.femsre.2004.12.005
Saitou, N., and Nei, M. (1987). The neighbor-joining method: a new method for reconstructing phylogenetic trees. Mol. Biol. Evol. 4, 406-425.

Skerman, V. B. D., McGowan, V., and Sneath, P. H. A. (eds). (1980). Approved lists of bacterial names. Int. J. Syst. Bacteriol. 30, 225-242. doi: 10.1099/00207713-30-1-225

Trapecar, M., Leouffre, T., Faure, M., Jensen, H. E., Granum, P. E., Cencic, A., et al. (2011). The use of a porcine intestinal cell model system for evaluating the food safety risk of Bacillus cereus probiotics and the implication for assessing enterotoxigenicity. APMIS 119, 877-884. doi: 10.1111/j.1600-0463.2011.02797.x

Williams, L. D., Burdock, G. A., Jiménez, G., and Castillo, M. (2009). Literature review on the safety of Toyocerin ${ }^{\circledR}$, a non-toxigenic and non-pathogenic Bacillus cereus var. toyoi preparation. Regul. Toxicol. Pharmacol. 55, 236-246. doi: 10.1016/j.yrtph.2009.07.009

Wilson, D. N. (2014). Ribosome-targeting antibiotics and mechanisms of bacterial resistance. Nat. Rev. Microbiol. 12, 35-48. doi: 10.1038/nrmicro 3155

Zwick, M. E., Joseph, S. J., Didelot, X., Chen, P. E., Bishop-Lilly, K. A., Stewart, A. C., et al. (2012). Genomic characterization of the Bacillus cereus sensu lato species: backdrop to the evolution of Bacillus anthracis. Genome Res. 22, 1512-1524. doi: 10.1101/gr.134437.111

Conflict of Interest Statement: The research was funded by Rubinum S.A. that has a commercial interest in B. toyonensis strain BCT-7112 ${ }^{\mathrm{T}}$. However, the other authors declare that the research was conducted in the absence of any commercial or financial relationships that could be construed as a potential conflict of interest.

The reviewer AJ declared a shared affiliation, though no other collaboration, with one of the authors $\mathrm{AB}$ to the handling Editor, who ensured that the process nevertheless met the standards of a fair and objective review.

Copyright (C) 2017 Glenwright, Pohl, Navarro, Miro, Jiménez, Blanch and Harwood. This is an open-access article distributed under the terms of the Creative Commons Attribution License (CC BY). The use, distribution or reproduction in other forums is permitted, provided the original author(s) or licensor are credited and that the original publication in this journal is cited, in accordance with accepted academic practice. No use, distribution or reproduction is permitted which does not comply with these terms. 